\title{
Development of an allergy management support system in primary care
}

\author{
Bertine MJ \\ Flokstra - de Blok ${ }^{1,2}$ \\ Thys van der Molen ${ }^{1,2}$ \\ Wianda A Christoffers ${ }^{3}$ \\ Janwillem WH Kocks ${ }^{1,2}$ \\ Richard L Oei ${ }^{4}$ \\ Joanne NG Oude Elberink ${ }^{2,4}$ \\ Emmy M Roerdink ${ }^{5}$ \\ Marie Louise Schuttelaar ${ }^{3}$ \\ Jantina $L$ van der Velde ${ }^{1,2}$ \\ Thecla M Brakel ${ }^{1,6}$ \\ Anthony EJ Dubois ${ }^{2,5}$ \\ 'Department of General Practice, \\ ${ }^{2}$ GRIAC Research Institute, \\ ${ }^{3}$ Department of Dermatology, \\ ${ }^{4}$ Department of Allergology, \\ ${ }^{5}$ Department of Pediatric \\ Pulmonology and Pediatric Allergy, \\ University of Groningen, University \\ Medical Center Groningen, \\ ${ }^{6}$ Teaching Unit, Department of Social \\ Psychology, University of Groningen, \\ Groningen, The Netherlands
}

Correspondence: Bertine MJ Flokstra de Blok

Department of General Practice, University Medical Center Groningen, Internal Postcode FA2I, PO Box 196, 9700 AD Groningen, The Netherlands Tel +3I $5036 \mid 6757$

Fax +3I 503632964

Email b.m.j.flokstra@umcg.nl
This article was published in the following Dove Press journal:

Journal of Asthma and Allergy

15 March 2017

Number of times this article has been viewed

Background: Management of allergic patients in the population is becoming more difficult because of increases in both complexity and prevalence. Although general practitioners (GPs) are expected to play an important role in the care of allergic patients, they often feel ill-equipped for this task. Therefore, the aim of this study was to develop an allergy management support system (AMSS) for primary care.

Methods: Through literature review, interviewing and testing in secondary and primary care patients, an allergy history questionnaire was constructed by allergists, dermatologists, GPs and researchers based on primary care and specialists' allergy guidelines and their clinical knowledge. Patterns of AMSS questionnaire responses and specific immunoglobulin E (sIgE)test outcomes were used to identify diagnostic categories and develop corresponding management recommendations. Validity of the AMSS was investigated by comparing specialist (gold standard) and AMSS diagnostic categories.

Results: The two-page patient-completed AMSS questionnaire consists of 12 (mainly) multiple choice questions on symptoms, triggers, severity and medication. Based on the AMSS questionnaires and sIgE-test outcome of 118 patients, approximately 150 diagnostic categories of allergic rhinitis, asthma, atopic dermatitis, anaphylaxis, food allergy, hymenoptera allergy and other allergies were identified, and the corresponding management recommendations were formulated. The agreement between the allergy specialists' assessments and the AMSS was 69.2\% (CI 67.2-71.2).

Conclusion: Using a systematic approach, it was possible to develop an AMSS that allows for the formulation of diagnostic and management recommendations for GPs managing allergic patients. The AMSS thus holds promise for the improvement of the quality of primary care for this increasing group of patients.

Keywords: allergy, development, diagnosis, management support system, primary care

\section{Background}

The prevalence of atopic allergies in the population is rising, and patients with allergies constitute a burgeoning problem for health care systems. Recently the European Academy for Allergy and Clinical Immunology (EAACI) Task Force for Allergy Management in Primary Care noted the unsustainability of the current model, in which the care for allergic patients is predominantly specialist based. ${ }^{1}$ The Task Force proposed a holistic primary care-based system where primary care should be equipped for caring allergic patients by receiving education and support from allergists. In addition, there should be clear guidelines for GPs about how to diagnose and manage patients with allergic diseases, including referral, when appropriate. ${ }^{1}$ 
In many areas in Europe, only very few allergists are available. ${ }^{2}$ Worldwide, the number of certified allergists per head of population ranges from 1:25 million to 1:16,000. ${ }^{3}$ In addition, referred patients usually need to wait $>6$ weeks for a consultation with an allergist. ${ }^{2}$ Although general practitioners (GPs) are well trained, few have sufficient knowledge of diagnosis and management of allergic patients., ${ }^{4,5}$ Moreover, GPs often experience difficulty in arriving at the correct diagnosis resulting in inadequate management. ${ }^{6}$

Although most GPs feel that they are generally able to manage patients with common allergies, there are specific issues that may need attention. In an earlier study, we found that GPs felt inadequately trained to manage food-related, insect-related and drug-related allergies as well as unfamiliar allergies, especially when anaphylactic reactions were involved (Brakel TM, et al, unpublished data, 2017). Furthermore, knowledge of skin-related problems was seen as suboptimal by the GPs themselves (Brakel TM, et al, unpublished data, 2017). In addition, GPs reported problems with the interpretation of specific immunoglobulin $\mathrm{E}$ (sIgE)-test results. Problems emerged when the patient's history suggested an allergy, but the sIgE-test results were not confirmative and conversely when positive sIgE-test results were found for allergens with no clinical relevance (Brakel TM, et al, unpublished data, 2017). Although GPs feel comfortable with handling asthma and rhinitis, there continues to be room for improvement, such as the recognition of allergic rhinitis $^{7}$ and the over-diagnosis of allergies (not confirmed by allergy specialists). ${ }^{8}$ In the UK, it has been estimated that a quarter and possibly half of allergy referrals to the hospital could probably be dealt with by a GP with special interest in allergy. ${ }^{9,10}$ Another issue is the under-prescription of epinephrine auto-injectors, especially, for children at risk for an anaphylactic reaction, ${ }^{11,12}$ which may be at least partly due to GPs not prescribing this medication to patients for whom it would be appropriate to do so. ${ }^{13}$

In the past, our group was successfully involved in the set-up and implementation of an asthma/COPD diagnostic support service, which showed high uptake $(11,000$ patients in 2015) and improved patients' outcomes. ${ }^{14}$ In order to support GPs with the diagnosis and management of allergic patients, we developed an allergy management support system (AMSS). To our knowledge, this is the first allergy support system for primary care based on a structured and extensive clinical history questionnaire and sIgE-test outcomes. In the past, only one partly comparable initiative has been undertaken by adding standardized sentences to sIgEtest outcomes. ${ }^{15}$ Our AMSS aims to help GPs to effectively cope with allergic patients in primary care practices. As the
AMSS may assist GPs to treat more patients in primary care, the system subsequently may help to reduce waiting times for specialist care by focusing scarce resources on those who most need secondary care.

\section{Methods}

The development of the AMSS was based on information obtained from the literature and from allergy specialists and GPs. In addition, semi-structured, in-depth interviews were conducted with 12 GPs (with no special interest in allergy), in order to obtain necessary information for the development of the AMSS that would make it fit for purpose and suitable for integration into the daily workflow of the GP (Brakel TM, et al, unpublished data, 2017). The research group, consisting of pediatric and adult allergists, dermatologists, GPs and allergy researchers, constructed a preliminary allergy history questionnaire based on primary care and specialists' allergy guidelines, and their clinical knowledge. This history questionnaire inquires about patients' symptoms relating to the different allergic disorders. Based on the patient's responses to this questionnaire as well as their sIgE-test outcomes, probable diagnoses and corresponding management recommendations are generated by the AMSS (Figure 1).

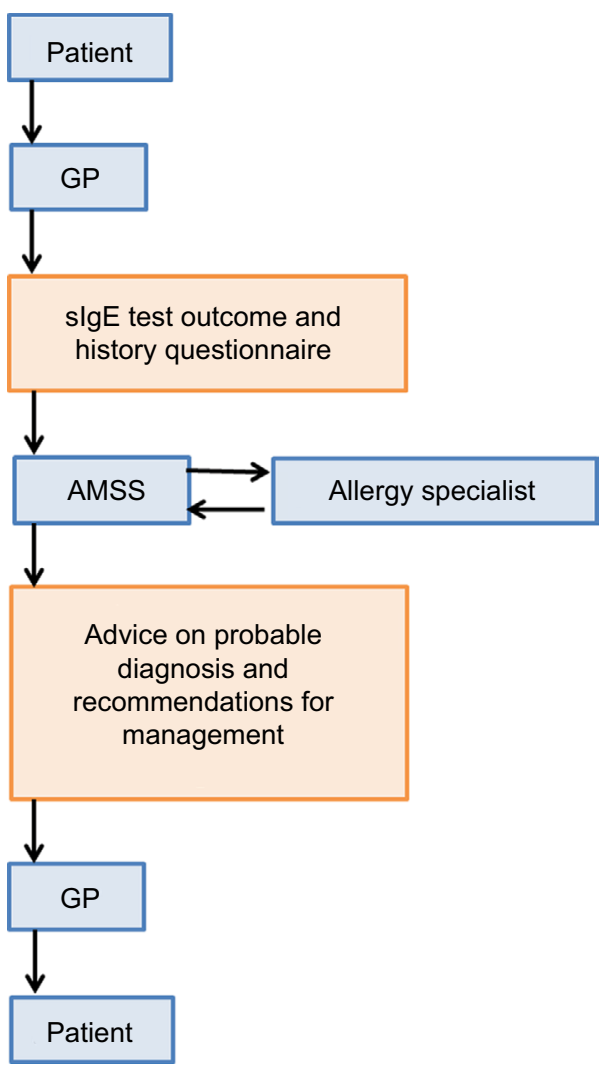

Figure I Schematic representation of the allergy management support system (AMSS).

Abbreviations: GP, general practitioner; slgE, specific immunoglobulin E. 


\section{Development of the AMSS questionnaire}

First, the history questionnaire was mock tested by allergy specialists and GPs. The allergy researcher completed five questionnaires with different hypothetical patients in mind. These questionnaires were analyzed by two allergy specialists and two GPs, and they individually formulated a diagnosis. Inconsistencies between the diagnosis of the researcher and the physicians were discussed within the research group. Amendments were made to the wording of certain questions in the questionnaire when needed.

Next, the history questionnaire was pre-tested in ten allergic patients and ten parents of allergic children. These subjects were asked to explain the meaning of each question in their own words. Based on possible difficulties or misunderstandings, further amendments were made to the wording of certain questions in the questionnaire. The resultant AMSS questionnaire was subject to several constraints: It had to be limited to two pages, with short, clear questions and multiplechoice answers using simple tick boxes.

\section{slgE testing by GPs}

In order to get more insights into what kind of allergic problems were seen in primary care as well as the reason for sIgE testing by GPs, we aimed at obtaining and analyzing 100 completed AMSS questionnaires. Primary care patients who were sent to the local GP laboratory for one or more $\operatorname{sigE}$ tests received the AMSS questionnaire. This questionnaire was self-completed at home by patients or their parents. At this stage, GPs did not receive recommendations from the AMSS, since the system was still under development.

\section{Development of the diagnostic classification and management recommendations}

The diagnostic categories, including disease severity (ie, mild, moderate, severe), were based on certain combinations of responses given to the AMSS questionnaire items (patterns). For each diagnostic category, corresponding management recommendations were formulated based on primary care and specialists' allergy guidelines complemented with clinical knowledge and expert opinion. Thus, with these response patterns, a rule-based system was developed that captures the relevant information from evidence-based medicine as well as knowledge of the allergy experts into distinct algorithms.

\section{Agreement between AMSS and specialist}

In order to validate the AMSS, the AMSS questionnaire was completed by patients who were referred by GPs to allergists (pediatric or adult) or dermatologists. The GPs who included these patients were not involved in this part of the study. Patients or their parents completed the questionnaire at home before their consultation with the specialist. The completed questionnaires were analyzed by two researchers (TMB [postdoc primary care] and EMR [medical student performing internship]) who allocated the patients to predefined diagnostic categories using the algorithms of the AMSS. Based on the clinical correspondence, two allergists (AEJD and RLO) and two dermatologists (MLAS and WAC) allocated the same patients to predefined diagnostic categories using their clinical knowledge, which was considered to be the gold standard (Figure 2). Agreement between the assessment by the specialist (gold standard) and the AMSS was investigated by ascertaining concordance of diagnostic categories in a qualitative and a quantitative way. In the qualitative analysis, agreement was categorized based on the diagnosis and degree of severity (mild, moderate and severe):

- Total agreement: same diagnosis and same severity;

- Substantial agreement: same diagnosis, but one severity degree apart;

- Partial agreement: same diagnosis, but more than one severity degree apart;

- No agreement: different diagnosis.

The quantitative analysis was restricted to the main allergic diagnostic categories (allergic rhinitis, asthma, atopic dermatitis, anaphylaxis, food allergy, hymenoptera allergy, latex allergy, work or hobby-related allergy, drug-related allergy and urticaria/angioedema), and the degree of severity

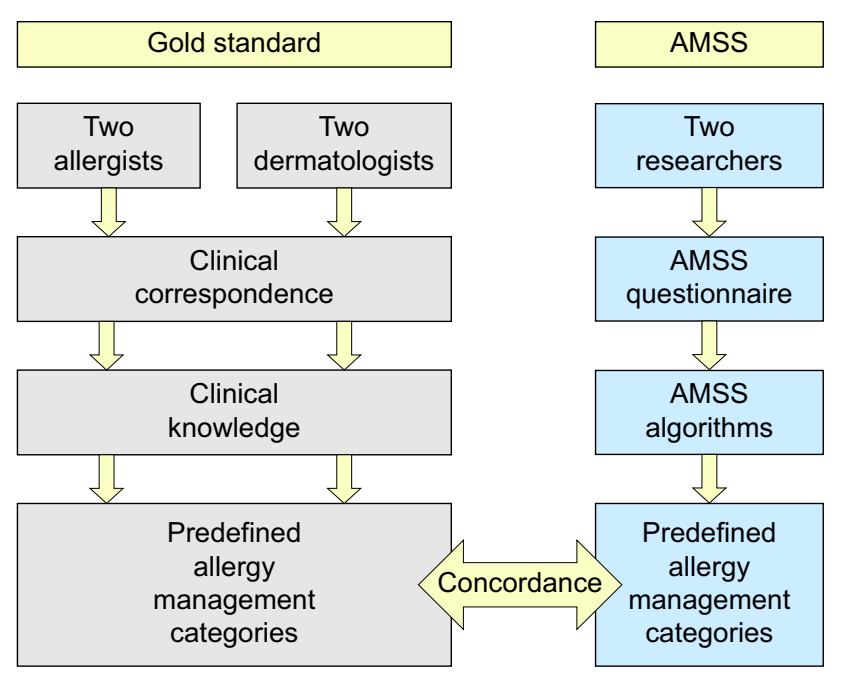

Figure 2 Flowchart of the validity study on agreement between the allergy specialist (gold standard) and the AMSS.

Abbreviation: AMSS, allergy management support system. 
was not taken into account. Only cases were used where there was agreement between specialists (gold standard) and also between researchers (AMSS). These constraints were imposed by the calculation of the agreement score. The agreement between the gold standard and AMSS was calculated as $(\mathrm{TP} / \mathrm{TP}+\mathrm{FN}) \times 100 \%$, where true positive $(\mathrm{TP})$ indicates that both the gold standard and the AMSS reported the same diagnosis and false negative $(\mathrm{FN})$ indicates that the AMSS missed a diagnosis made by the gold standard. The resultant percentage indicates the percentage of correct diagnoses from the AMSS. The inter-rater agreement between the allergists, dermatologists and researchers was investigated using Cohen's Kappa.

This study was approved by the local medical ethics committee of the University Medical Center Groningen (METc 2011/273) who deemed that the study did not fall within the Medical Research Involving Human Subjects Act. Official informed consent from participants was therefore not required. Participation was voluntary, and all participants received written and/or oral information about the study.

\section{Results}

\section{Description of the content of the AMSS questionnaire}

The AMSS questionnaire contains questions relating to the different allergic disorders. It includes questions relating to the diagnosis as well as disease severity and whether a doctor's diagnosis has been made. Current medications and their effectiveness were documented in order to assess whether the medication prescribed was in keeping with the severity of the disease. Questions about possible environmental and food allergens were included as well as circumstances and consequences of exposure, including exposures at work (Figure S1).

\section{Allergic (seasonal and perennial) rhino conjunctivitis Questions included symptom type and severity (based on Control of Allergic Rhinitis and Asthma Test), ${ }^{16}$ presence and degree of seasonality, and effect of medication. The goal was to identify severity and symptom control with pharmacotherapy (including self-reported compliance) as well as suitability for immunotherapy with pollen, house dust mite or cat.}

\section{Asthma}

Questions included the type and severity of symptoms and secondary impact on daily activities (based on Control of Allergic Rhinitis and Asthma Test). ${ }^{16}$ Medications and self-reported compliance were included in the assessment.
The effect of both allergic and non-allergic triggers was also covered.

\section{Atopic dermatitis}

Questions addressed the age of onset, distribution of clinical signs, family history of atopic disease and the presence of a dry skin (based on the United Kingdom Working Party's Diagnostic Criteria for Atopic Dermatitis). ${ }^{17}$ Additional questions addressed the severity of symptoms and recent topical treatment. With regard to severity and treatment, separate questions were posed about the face and the rest of the body.

\section{Anaphylaxis}

Symptoms of anaphylaxis were recorded as well as the suspected cause, including foods, drugs, latex, insect stings or none of these causes. A question distinguishing local from systemic reactions to insect stings was included. Based on the type of symptoms that occurred, high-risk patients could be distinguished from low-risk patients, and the need for an epinephrine auto-injector could be assessed. High-risk food allergy was defined when at least one of the following criteria was met: 1) previous history of a life-threatening anaphylactic reaction to a food; 2) previous asthmatic reaction caused by a food; 3) severe (uncontrolled) asthma; 4) any two of the following: a) aged $>12$ years; b) coexistent asthma; c) suspected allergy to peanut, nuts, sesame, crustaceans or fish; and d) previous clear systemic reaction to traces of food (derived from the EAACI guideline for anaphylaxis). ${ }^{18}$

\section{Food allergy}

Apart from the suspected food, circumstances surrounding ingestions resulting in reactions were documented, including the time between ingestion and reaction, estimated quantity ingested and duration of symptoms. The effect on symptoms of dietary elimination of the allergenic food was recorded. A separate question addressed tolerance to milk products other than milk itself in patients with suspected allergy to cow's milk.

\section{slgE testing by GPs}

In total, 118 primary care patients completed the AMSS questionnaire. The mean age was 31.7 years (range from 12 months to 81 years), and $40 \%$ was male. The following were indications for sIgE testing in these patients: rhinitis (51\%), asthma (26\%), eczema (15\%), food allergy $(9 \%)$, anaphylaxis (1\%) and others (18\%). The questionnaire appeared to be user-friendly since the vast majority of the questionnaires were correctly completed. 


\section{Description of the diagnostic classification and management advice}

The combination of the patient's responses to the AMSS questionnaire with the sIgE-test outcomes allowed for allocation of patients to distinct diagnostic categories. Each diagnostic category was coupled to a management recommendation. In total, there are currently approximately 150 different categories of allergic rhinitis, asthma, atopic dermatitis, anaphylaxis, food allergy, hymenoptera allergy and other allergies (latex allergy, work-related allergy, drug-related allergy and urticaria). These categories were based on the type of allergy, severity and treatment status. Also, non-allergic categories were defined (eg, "non allergic rhinitis" and "unlikely food allergy"). The management recommendations were usually structured as an initial therapeutic option, followed by subsequent management steps to be followed if the initial treatment did not have the desired effect. The management recommendations could also include non-therapeutic recommendations and referral recommendations (Box 1). Background information accompanying the recommendations was provided to the GP as an appendix. This information was specifically formulated for the AMSS.

\section{Agreement between AMSS and specialist}

The AMSS questionnaire was completed by 42 patients or their parents who had been referred by GPs to allergists (pediatric or

Box I Example of AMSS recommendations for a GP.

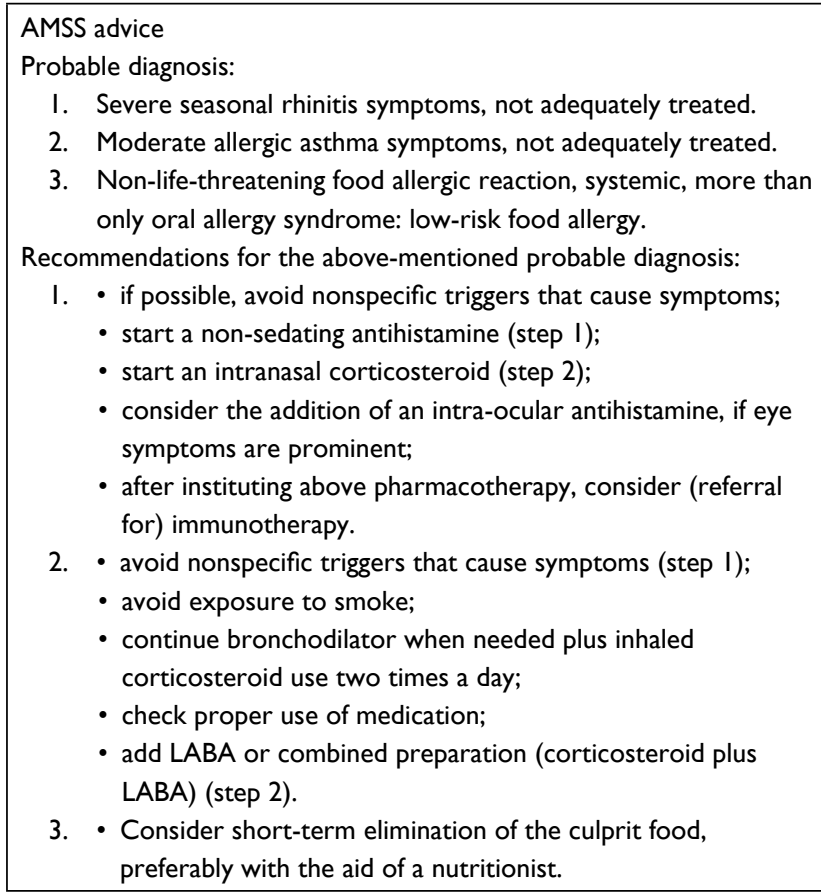

Abbreviations: AMSS, allergy management support system; GP, general practitioner LABA, long-acting bronchodilator. adult) or dermatologists. The majority of patients were female (62\%) with a mean age of 26 years (range from 2 months to 68 years). The mean time between completing the questionnaire and the consult was 8 days (SD 11 days). About half of them consulted the allergist $(n=20)$ and the others consulted the pediatric allergist $(n=11)$ or dermatologist $(n=11)$. The qualitative agreement (sum of total, substantial and partial agreement) between the specialists (gold standard) and the researchers (AMSS) ranged between $54 \%$ and $87 \%$. The quantitative agreement between the gold standard and the AMSS was $69 \%$ (CI 67-71). This indicates the percentage of correct diagnoses made by the AMSS. The inter-rater agreement between the two allergists was moderate $(k=0.55$, CI $0.37-0.72)$, as was the inter-rater agreement between the two dermatologists $(k=0.53$, CI 0.14-0.91). The inter-rater agreement between the two researchers was high $(k=0.98$, CI 0.94-1.01).

\section{Discussion}

\section{Main findings}

The AMSS presented here is the first support system for allergy management in primary care and may be a useful tool for GPs, allowing them to provide quality care for allergic patients. It covers a wide variety of allergic diseases, and yet only comprises a two-page questionnaire and sIgE-test results. There was good agreement between the AMSS and specialist recommendations (gold standard), supporting the validity of the AMSS.

\section{Interpretation of the findings in relation to previously published work}

The AMSS was inspired by the Asthma COPD service (ACservice), ${ }^{14}$ which allows GPs to refer patients with possible obstructive airway disease to the AC-service. Patients complete a history questionnaire, and spirometry is performed at the GP laboratory. Pulmonologists assess these patient data through an online secured internet portal and send a diagnosis and management recommendation to the GP. Currently, 11,000 patients have been assessed by the AC-service, and it is considered to be feasible and effective. ${ }^{14}$ Comparable outcomes may be expected for the AMSS when it is implemented in a broader fashion. However, an important difference between the AMSS and the Asthma COPD service is that specialists involved in the $\mathrm{AC}$-service assess all patient information individually, whereas in the AMSS patient information assessment will ultimately be automated as much as possible.

An important aspect of the functioning of the AMSS is that it will probably have a learning effect. GPs are likely to become more effective in diagnosing, managing and referring 
allergic patients ${ }^{9}$ when working with the recommendations of the AMSS. GPs will probably develop a better understanding of whether sIgE determinations are required and how they should be interpreted. This direct patient-related learning might be one of the benefits of the AMSS and may, in general, improve care for patients with allergies. ${ }^{19}$ This aspect of the AMSS is important because it is well recognized that knowledge of guidelines is generally poor, and that such guidelines are generally not followed..$^{20,21}$

A further possible advantage of the AMSS may be that it does not just focus on the patient's main reason for seeking consultation. Because of the comprehensive nature of the AMSS questionnaire, it could also address comorbid allergic disorders. This is particularly relevant in primary care where GPs are often limited to a single medical problem because of time constraints, while multiple atopic conditions are frequently present in the same patient. ${ }^{22}$

Finally, it should be noted that the AMSS questionnaire is not intended to replace a specialist consultation but rather to support GPs in diagnosing and managing patients with allergic symptoms. The AMSS generates recommendations based on the answers on the AMSS questionnaire and the sIgE-test results. The AMSS recommendations empower the GP to manage many patients with allergic symptoms in primary care, thus avoiding unnecessary referrals. In complex or high-risk cases, the AMSS advises the GP to refer the patient to a specialist. Ultimately, GPs remain responsible and autonomous, and are free to deviate from the recommendations of the AMSS.

Although previous studies (Brakel TM, et al, unpublished data, 2017) ${ }^{6}$ show that GPs felt more comfortable managing patients with airway allergy (ie, asthma and rhinitis) than those with systemic allergy (ie, food allergy and anaphylaxis), we found that $\operatorname{sigE}$ tests were often requested for both the types of allergy. Therefore, the AMSS includes information on the proper interpretation of sIgE-test results for both airway allergy and systemic allergy.

\section{Strengths and limitations of this study}

A strength of this study is the comprehensive approach to develop the AMSS and its content. Multiple steps and different approaches were used to develop and fine-tune the AMSS questionnaire and the resultant diagnostic classification and management advice. A limitation of this study at this point in time is the number of patients who completed the AMSS questionnaire in relation to the different diagnostic categories. However, many patients have comorbid allergic disorders, resulting in more diagnostic categories than patients. Thus, most diagnostic categories are covered by patients, and this may be considered sufficient to proceed with further utilization of the AMSS.

\section{Conclusion}

This is the first allergy management support system designed for use in primary care. We showed that it was possible to develop an AMSS that allows for the formulation of diagnostic and management recommendations for GPs managing allergic patients. Our results support the validity of this AMSS. The AMSS thus holds the promise for improvement of the quality of primary care for allergic patients.

\section{Recommendations}

In the future, the AMSS will be computerized and further tested and validated in the primary care setting. In an automated form, the AMSS can be run efficiently within existing systems, allowing GPs to provide up-to-date high-quality management ${ }^{23}$ and may improve selection of patients for specialist referral.

\section{Acknowledgments}

This work was supported by unrestricted grants from Phadia BV (2009) and ALK Abelló BV (2013-12ACT-05). These funding bodies were not involved in designing the study, nor the collection, analysis and interpretation of data and writing the manuscript.The authors would like to thank the GPs and Certe (local GP laboratory) for distributing the AMSS questionnaire to patients in the developmental phase of the AMSS.

\section{Author contributions}

All authors contributed to conception and design, acquisition of data, or analysis and interpretation of data, drafting the article or revising it critically for important intellectual content, gave final approval of the version to be published, and agree to be accountable for all aspects of the work.

\section{Disclosure}

The authors report no conflicts of interest in this work.

\section{References}

1. Jutel M, Angier L, Palkonen S, et al. Improving allergy management in the primary care network-a holistic approach. Allergy. 2013; 68(11):1362-1369.

2. Agache I, Ryan D, Rodriguez MR, Yusuf O, Angier E, Jutel M. Allergy management in primary care across European countries - actual status. Allergy. 2013;68(7):836-843.

3. Warner JO, Kaliner MA, Crisci CD, et al; World Allergy Organization Specialty and Training Council. Allergy practice worldwide: a report by the World Allergy Organization Specialty and Training Council. Int Arch Allergy Immunol. 2006;139(2):166-174.

4. Hazeldine M, Worth A, Levy ML, Sheikh A. Follow-up survey of general practitioners' perceptions of UK allergy services. Prim Care Respir J. 2010;19(1):84-86, 7p following 86. 
5. Lowe G, Kirkwood E, Harkness S. Survey of anaphylaxis management by general practitioners in Scotland. Scott Med J. 2010;55(3):11-14.

6. Gupta RS, Springston EE, Kim JS, et al. Food allergy knowledge, attitudes, and beliefs of primary care physicians. Pediatrics. 2010;125(1):126-132.

7. Ryan D, van Weel C, Bousquet J, et al. Primary care: the cornerstone of diagnosis of allergic rhinitis. Allergy. 2008;63(8):981-989.

8. Kaminski ER, Bethune CA, Jones RB. Complexity of case mix in a regional allergy service. BMC Res Notes. 2012;5:103.

9. Smith HE, Wade J, Frew AJ. What proportion of adult allergy referrals to secondary care could be dealt with in primary care by a GP with special interest? Clin Transl Allergy. 2016;6:3.

10. Conlon NP, Abramovitch A, Murray G, et al. Allergy in Irish adults: a survey of referrals and outcomes at a major centre. Ir J Med Sci. 2015; 184(2):349-352.

11. Flokstra-de Blok BM, Doriene van Ginkel C, Roerdink EM, et al. Extremely low prevalence of epinephrine autoinjectors in high-risk foodallergic adolescents in Dutch high schools. Pediatr Allergy Immunol. 2011;22(4):374-377.

12. Calamelli E, Mattana F, Cipriani F, Ricci G. Management and treatment of anaphylaxis in children: still too low the rate of prescription and administration of intramuscular epinephrine. Int J Immunopathol Pharmacol. 2014;27(4):597-605.

13. Saleh-Langenberg J, Dubois AE, Groenhof F, Kocks JW, van der Molen T, Flokstra-de Blok BM. Epinephrine auto-injector prescriptions to foodallergic patients in primary care in The Netherlands. Allergy Asthma Clin Immunol. 2015;11:28.

14. Metting EI, Riemersma RA, Kocks JH, Piersma-Wichers MG, Sanderman R, van der Molen T. Feasibility and effectiveness of an asthma/ COPD service for primary care: a cross-sectional baseline description and longitudinal results. NPJ Prim Care Respir Med. 2015;25:14101.
15. de Groot H, Lous J, Peters L, Sandell A, Onell A. An allergy decision support system for non-allergy specialists: test and evaluation in routine. Allergy. 2009;64(Suppl 90):533.

16. van der Leeuw S, van der Molen T, Dekhuijzen PN, et al. The minimal clinically important difference of the control of allergic rhinitis and asthma test (CARAT): cross-cultural validation and relation with pollen counts. NPJ Prim Care Respir Med. 2015;25:14107.

17. Williams HC, Burney PG, Hay RJ, et al. The U.K. Working Party's Diagnostic Criteria for Atopic Dermatitis. I. Derivation of a minimum set of discriminators for atopic dermatitis. Br J Dermatol. 1994;131(3) 383-396.

18. Muraro A, Roberts G, Worm M, et al; EAACI Food Allergy and Anaphylaxis Guidelines Group. Anaphylaxis: guidelines from the European Academy of Allergy and Clinical Immunology. Allergy. 2014; 69(8):1026-1045.

19. Otto AK, Dyer AA, Warren CM, Walker M, Smith BM, Gupta RS. The development of a clinical decision support system for the management of pediatric food allergy. Clin Pediatr (Phila). Epub 2016 Oct 26.

20. Warner JO. Do we need more management guidelines? Pediatr Allergy Immunol. 2007;18(7):549-550.

21. Lugtenberg M, Zegers-van Schaick JM, Westert GP, Burgers JS. Why don't physicians adhere to guideline recommendations in practice? An analysis of barriers among Dutch general practitioners. Implement Sci. 2009; $4: 54$

22. Pols DH, Wartna JB, van Alphen EI, et al. Interrelationships between atopic disorders in children: a meta-analysis based on ISAAC questionnaires. PLoS One. 2015;10(7):e0131869.

23. Matui P, Wyatt JC, Pinnock H, Sheikh A, McLean S. Computer decision support system for asthma: a systematic review. NJP Prim Care Respir Med. 2014;24:14005. 


\section{Supplementary material}

Patient name...

Date of birth of the patient...

Date of completion...
Name of general practitioner...

Sex of patient: male/female

Social security number..

1. What is the reason for this allergy investigation according to you?...

2. On average, how often do you have the following symptoms during the period of the year during which you are most severely affected?

- Never/sometimes/1 or 2 days a week/more than 2 days a week/(almost) every day

2.1 Blocked nose

2.2 Sneezing/itchy nose

2.3 Itchy and/or tearing eyes

2.4 Runny nose

2.5 Shortness of breath/dyspnea

2.6 Coughing

2.7 Wheezing in the chest

2.8 Chest tightness upon physical exercise

2.9 Limitations in doing daily tasks because of Nasal symptoms Chest symptoms

2.10 Waking up during the night because of Nasal symptoms Chest symptoms Other, namely...

2.11 During the period of the year during which you are most severely affected, how often did you have to increase your medications because of nasal symptoms and/or asthma?

- Never/sometimes/daily

2.12 Outside the period of the year during which you are most severely affected the symptoms are

- The same/less severe/much less severe/absent

3. Which of the following is present in your home?

- Cat/dog/bird/rodent/mold/textile floor covering in bedroom

4.1 Has a doctor ever diagnosed one or more of the following?

- Asthma/hay fever/eczema/food allergy/other allergy/l do not know/not diagnosed

4.2 From which of the aforementioned disease(s) do you have currently symptoms?

- Asthma/hay fever/eczema/food allergy/other allergy/l do not know

5. Last year, did you have an itchy skin disease?

- Yes, go to question 5.1, 5.2, 5.3, 5.4, and 6. No, skip question 5.1, 5.2, 5.3, 5.4, and 6 .

5.1 At which age did this skin disease start?

- Before the 2nd year/2-4 years/5-10 years/later

5.2 Has this skin disease ever been present in the inside of the elbows, back of the knees, front of ankles, neck or around the eyes?

- Yes/no/l do not know

5.3 Last year, did you generally have dry skin?

- Yes/no/l do not know

5.4 Do one of your parents, brother or sisters have hay fever, asthma or eczema?

- Yes/no/l do not know

6. Complete question 6.1 and 6.2 if you have eczema

6.1 How severe was the eczema last week?

- Face $>$ not $/$ mild/moderate/severe/very severe

- Body $>$ not $/$ mild $/$ moderate/severe/very severe

How burdensome was the eczema last week?

- Face $>$ not/a little/moderately/much/very much

- Body $>$ not/a little/moderately/much/very much

6.2 Last week, which ointments/creams have been used for eczema and how often?

On the face; $\ldots$

On the body;...

7. Do you have allergic symptoms when you come into contact with:

- Food(s)/wasp and/or honeybee sting/medication/latex/unknown/no $\rightarrow$ go to question 8

7.1 If symptoms occur, which symptoms are they? (multiple answers possible)

- Dizziness and/or palpitations; unconsciousness; wheezing and/or dyspnea; feeling of throat tightening; vomiting and/or diarrhea; nausea and/or stomach cramps; swollen tongue and/or lips; nasal symptoms; itchy mouth, ears and/or throat; itchy tongue and/or lips; itchy and/or tearing eyes; worsening eczema (one spot/many spots/all over body); itchy skin (one spot/many spots/all over body); red rash (one spot/many spots/all over body); swelling of the skin (one spot/many spots/all over body); hives (one spot/many spots/all over body); other symptoms, namely....

Figure SI (Continued) 
7.2 In case of wasp and/or honeybee sting, where do the aforementioned skin symptoms occur?

- At the place of sting/elsewhere

8.1 During the last 3 months, did you have hives?

- Yes, some/yes, quite a few/yes, a lot/no

8.2 During the last 3 months, how often did you have hives?

- Daily/weekly/monthly/not at all

9. Are the allergic symptoms possibly related to your work or hobby?

- Yes/no/l do not know

10. Please indicate when or which trigger causes allergic symptoms (multiple answers possible)

- Spring/summer/fall/winter/exercise/fog/cold air/cigarette smoke/paint fumes/perfume fumes/cooking fumes/ grasses/trees/dog/cat/horse/(house) dust/weeds/cow's milk*/egg/soy (milk)/apple/sesame/peanut/almond/walnut/ cashew/hazelnut/pistachio nut/fish (1 or more species)/shell fish (1 or more species)/latex and or natural rubber/ medicine/wasp/honeybee venom/l do not know/other, namely.....

${ }^{*}$ In case of cow's milk, can you eat one of the following products without symptoms; cheese, Biogarde ${ }^{\circledR}$, yogurt, custard, Danoontje ${ }^{\circledR}$ and/or goat's milk?

- Yes/no/l do not know

11. Do symptoms occur upon eating certain foods?

- Yes/no (go to question 12)

11.1 If symptoms occur upon eating certain foods, please indicate how quickly they occur?

- After a few minutes to an hour/after a few hours/after a day or longer/l do not know

If symptoms occur upon eating certain foods, please indicate the amount that causes them?

- Traces/crumbs to a few bites or swallows/daily serving to more than a daily serving/l do not know

If symptoms occur upon eating certain foods, please indicate how long they persist?

- A couple of hours/a day or longer/a week or longer/l do not know

11.2 When there is no exposure to the food that (possibly) causes the symptoms, are there.....?

- No allergic symptom/l do not know/sometimes there are allergic symptoms, namely....

12. Do you use medications for your allergic symptoms or asthma (including over the counter medications)?

- No/yes, which?

Medicine name: ...

Type of administration

- Tablet/inhaler/injection/adrenaline or EpiPen/syrup or solution/suppository/nasal spray/eye drops

Dose: ...

How often used?...

How well does it work?

- It does not work/inadequate effect/adequate effect/very adequate effect

Please check whether all applicable questions are completed. Thank you very much!

Figure SI Content of the provisional version of the allergy management support system questionnaire.

Note: This is an English translation of the original questionnaire, which was in Dutch. The content of the questionnaire has been retained but the formatting has been changed.

Journal of Asthma and Allergy

\section{Publish your work in this journal}

The Journal of Asthma and Allergy is an international, peer-reviewed open access journal publishing original research, reports, editorials and commentaries on the following topics: Asthma; Pulmonary physiology; Asthma related clinical health; Clinical immunology and the immunological basis of disease; Pharmacological interventions and

new therapies. This journal is included in PubMed. The manuscript management system is completely online and includes a very quick and fair peer-review system, which is all easy to use. Visit http://www. dovepress.com/testimonials.php to read real quotes from published authors. 